\title{
USE OF RETROSPECTIVE OPTIMIZATION FOR PLACEMENT OF OIL WELLS UNDER UNCERTAINTY
}

\author{
Honggang Wang \\ David Echeverría Ciaurri \\ Louis J. Durlofsky \\ Department of Energy Resources Engineering \\ Stanford University \\ Stanford, CA 94305-2220
}

\begin{abstract}
Determining well locations in oil reservoirs under geological uncertainty remains a challenging problem in field development. Well placement problems are integer optimization problems because a reservoir is discretized into grid blocks and the well locations are defined by block indices $(i, j, k)$ in the discrete model. Reservoir simulators are used to evaluate reservoir production given a well placement. In the presence of reservoir uncertainty, we simulate multiple model realizations to estimate the expected field performance for a certain well placement. Most existing methods for well placement optimization problems are random-search based algorithms.

We present a retrospective optimization (RO) algorithm that uses Hooke-Jeeves search for well location optimization under uncertainty. The RO framework generates a sequence of sample-path problems with increasing sample sizes. Embedded in RO, the Hooke-Jeeves search solves each sample-path problem for a local optimizer given a discrete neighborhood definition. The numerical results show that the $\mathrm{RO}$ algorithm efficiently finds a solution yielding a 70\% increase (compared to a solution suggested from heuristics) in the expected net present value (NPV) over 30 years of reservoir production for the problem considered.
\end{abstract}

\section{INTRODUCTION}

Geological formations, including reservoirs that contain oil, typically display highly variable physical properties. The key property that impacts fluid flow is the rock permeability (essentially a conductivity measure relating flow rate to pressure gradient), which can vary by many orders of magnitude over the formation. Other reservoir properties may also show considerable spatial variation. As a result of this variability, well performance (e.g., rate of oil production, fraction of oil in produced fluid) can vary significantly over the reservoir. Because well costs can be extremely high, particularly in offshore settings, it is essential that wells be drilled in productive locations. The problem is even more challenging as the formation properties are typically highly uncertain.

Reservoir flow simulation is widely used for guiding decisions regarding oil reservoir development and management. Reservoir simulators represent oil reservoirs in terms of discrete grid blocks and then solve numerically the partial differential equations governing subsurface flow (analytical solutions are not possible for practical problems). These simulations can be time consuming as the problem is transient, and realistic models may contain $O\left(10^{4}-10^{6}\right)$ grid blocks, with multiple unknowns per block (the actual number depends on the system under study). The expected reservoir performance under geological uncertainty can be estimated by running simulations on multiple realizations of the system.

Well location optimization can be accomplished using the reservoir simulator to evaluate a specified objective function. This is a discrete optimization problem in which we seek to determine well locations in the discretized model; i.e., on a grid. The decision variables correspond to a vector of positive integers that define the centers of reservoir blocks where wells are located. Stochastic optimization approaches are the most widely used techniques for this problem, though they may present 
slow convergence. See, e.g., Yeten (2003), Onwunalu and Durlofsky (2010), Sarma and Chen (2008) and Bangerth et al. (2006). In addition, although some investigators have incorporated geological uncertainty in the optimizations, there does not yet appear to be a computationally efficient means of optimizing under uncertainty.

In this paper, we present a new procedure for optimizing the locations of new wells under uncertainty. The procedure entails a discrete Hooke-Jeeves (HJ) (Hooke and Jeeves 1961) search algorithm embedded in a retrospective optimization (RO) framework. HJ search is a gradient-free direct search algorithm that seeks improved solutions in coordinate and pattern directions. Jin and Schmeiser (2003) presented an RO framework for continuous stochastic optimization. Wang and Schmeiser (2008) extended the continuous RO to discrete retrospective optimization (DRO). RO algorithms generate a sequence of sample-path problems with the sample sizes asymptotically increasing to infinity. The objective function of each sample-path problem is approximated by the sample average of the system performance. The sequence of such sample-path objective functions converges to the true objective function in the original problem as the sample size approaches infinity. In the RO framework, the initial solution for the current sample-path problem is simply the returned solution from the previous sample-path problem. In early sample-path iterations, $\mathrm{RO}$ algorithms do not require excessive computation because the sample sizes are small. In late iterations, RO algorithms are still reasonably efficient because the initial solutions are (expected to be) closer to the optimum.

This paper is structured as follows. In the next section we introduce the main well location optimization problem. The retrospective optimization algorithm is described in Section 3, and applied to a reservoir model in Section 4.

\section{PROBLEM STATEMENT}

We seek to optimize well placement using reservoir simulation. The decision variables are positive integers that specify the locations of grid blocks containing wells. Only vertical wells are considered here, and wells are assumed to be centered in the grid blocks they penetrate. The objective function, which could be the expected net present value (NPV) or the total amount of oil produced, is stochastic as a result of uncertainty in the geological properties of the underlying reservoir model.

We denote $N(x)$ as the neighborhood of a solution $x$. In this paper, we define the discrete neighborhood $N(x)=\left\{y \in \mathbb{Z}^{d}:\|y-x\|_{2} \leq 1\right\}$ for any $x \in \mathbb{Z}^{d}$, where \|\|$_{2}$ represents the Euclidean norm of a vector and $\mathbb{Z}^{d}$ is the set of $d$-dimensional integer vectors. With this neighborhood definition, $N(x)$ contains the $d+1$ closest integer points (including $x$ ) to $x$ in $\mathbb{Z}^{d}$. For a discrete optimization problem, we say a feasible solution is a local maximizer of the objective function, if the solution has the greatest objective function value compared to the feasible solutions in $N(x)$. With this neighborhood definition, the class of problems of interest can be mathematically formulated as follows.

$(P)$ Find a solution $x^{*}$ that locally maximizes $g=E[G(x, \quad)]$, where $E$ represents the expectation function, $x \in \mathbb{X} \subset \mathbb{Z}^{d}$, with $\mathbb{Z}^{d}$ the set of $d$-dimensional integer vectors, represents the stochastic effects in $G$, and $G$ is a simulation oracle that returns the sample observation of $g$ for a given $x$ and one realization of . The set $\mathbb{X}$ is assumed to be bounded (thus the problem has a finite number of feasible solutions).

In the current context, $g$ is the expected NPV over a certain production time and $G(x, \quad)$ is a stochastic function that calls the reservoir simulator to calculate the oil production and NPV for a given well placement $x$. Here $x$ is a vector of well locations $\left(i, j, k_{1}, k_{2}\right)$, explained below, and the random vector specifies the set of realizations of the reservoir model constructed using available data and geostatistical tools. In oil field development, geologists usually generate a finite set of such model realizations. Thus $g$ is the average of a finite number of observations through $G$.

In our numerical study, we use 104 realizations that were generated based on a North Sea reservoir. This (synthetic) model is referred to as the Brugge reservoir (Peters et al. 2009). We apply the RO algorithm to find well locations that locally maximize the average NPV over the 104 realizations. Given more realizations, the objective function $g$ could be the system performance averaged over a much larger number of realizations.

\section{RETROSPECTIVE OPTIMIZATION ALGORITHM USING HOOKE-JEEVES SEARCH}

Retrospective optimization (RO) (Chen and Schmeiser 2001) solves a sequence of sample-path optimization problems where the true objective function is approximated by the average of selected 
samples. We present an RO algorithm using the Hooke-Jeeves search (ROHJ) as the deterministic optimizer for all constructed sample-path problems. The ROHJ algorithm is described as follows.

Given: a bounded feasible region $\mathbb{X}$, an initial solution $x_{0} \in \mathbb{X}$, a simulation oracle $G$, an increasing sequence of sample sizes $m_{1}, m_{2}, \ldots$, a sequence of stochastic inputs $1,2, \ldots$, and a sequence of the initial step sizes $s_{1}, s_{2}, \ldots$ for the $\mathrm{HJ}$ algorithm.

Return: A local maximizer $x^{*}$ of $g$.

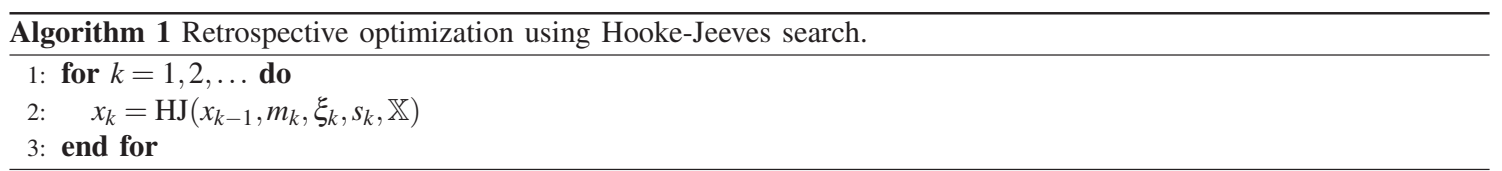

The RO framework first generates a sequence of sample-path problems $P_{k}, k=1,2, \ldots$. As $k$ increases, the problem $P_{k}$ approaches the true problem $P$. ROHJ is a specific implementation of $\mathrm{RO}$ algorithms that uses HJ direct search as the deterministic optimization tool for every samplepath problem $P_{k}$. Within the $k^{\text {th }}$ sample-path iteration, HJ solves $P_{k}$ for a local optimizer $x_{k}$ of $\bar{G}\left(x, m_{k}, k\right)=\frac{1}{m_{k}} \quad m_{k=1}^{m_{k}} G(x, i)$, where $m_{k}$ is the sample size and $\quad k=\left(\begin{array}{l}1,2 \\ i=1, m_{k}\end{array}\right)$ are the $m_{k}$ random inputs for $P_{k}$. If $g$ is the average over an infinite sample population, then as $k \rightarrow$ the sample-path solution $x_{k}$ asymptotically approaches a local maximizer $x^{*}$ of $g$ (Kleywegt et al. 2001). If $g$ is the mean over a finite population, then $x_{K}$ is a local maximizer of $g$ returned from the $K^{t h}$ sample-path problem, where $K$ samples the whole population.

The feasible region $\mathbb{X}$ for well location optimization problems is mainly constrained by the reservoir boundary. This particular structure of the feasible region allows some pattern search algorithms, such as HJ, to perform well. In our numerical experiments, we have observed that HJ efficiently finds local optimizers $x_{k}$ for every $P_{k}$, and eventually a better solution to the original problem $P$.

$\mathrm{HJ}$ is a standard implementation of the Hooke-Jeeves direct search modified for integer optimization. The sample average $\bar{G}(x, m, \quad)$ is calculated through the reservoir simulation $G$ for any feasible solution $x$, where the sample size $m$ specifies the number of model realizations and defines these $m$ realizations of the reservoir model. We denote the $d$-dimensional unit vector $e_{j}=\{0,0, \ldots, 1, \ldots, 0\}$ with the $j^{\text {th }}$ component being 1 . Such a unit vector $e_{j}$ represents the $j^{\text {th }}$ coordinate direction and is iteratively used by the exploratory search in HJ. As a discrete search algorithm, HJ restricts the step sizes to be positive integers. The sequence of step sizes $s$ for every $P_{k}$ is a geometric sequence (rounded to be integers) with the common ratio $1 / 2$. When the search approaches a local optimizer, HJ shrinks its search region by reducing the step size $s$ until $s=1$, when a local optimizer is returned.

As presented in Algorithm 2, HJ is simply a loop of direct search (gradient-free optimization) that alternates an exploratory search and a pattern move. The exploratory (coordinate-wise) search in $\mathrm{HJ}$ returns the best solution within local regions. When the step size reaches one, if the exploratory search evaluates the solutions in the neighborhood $N(x)$ of the current candidate solution $x$ and finds no improvement, then a local maximizer is returned. The pattern move follows the direction from the previous exploratory search and jumps to a (hopefully) better solution. When HJ finds an infeasible solution, the Project function projects the solution back to $\mathbb{X}$. Depending on $\mathbb{X}$, the Project function can be a simple component-wise projection onto the boundary of $\mathbb{X}$ or it can involve nonlinear constraints, such as well-to-well distance constraints.

Given a finite $\mathbb{X}, \mathrm{HJ}$ always finds a local optimum in finite computing time for every $P_{k}$, which ensures the termination and convergence of HJ. The finite solution time for every $P_{k}$, in turn, guarantees the local convergence of ROHJ.

The HJ algorithm is described as follows.

Given: A feasible integer region $\mathbb{X}$, initial solution $x_{0} \in \mathbb{X}$, initial integer step size $s \geq 1$, a vector of random inputs, and the sample size $m$.

Find: A local maximizer $x^{*}$ of $\bar{G}(x, m, \quad)$ over $\mathbb{X}$. 


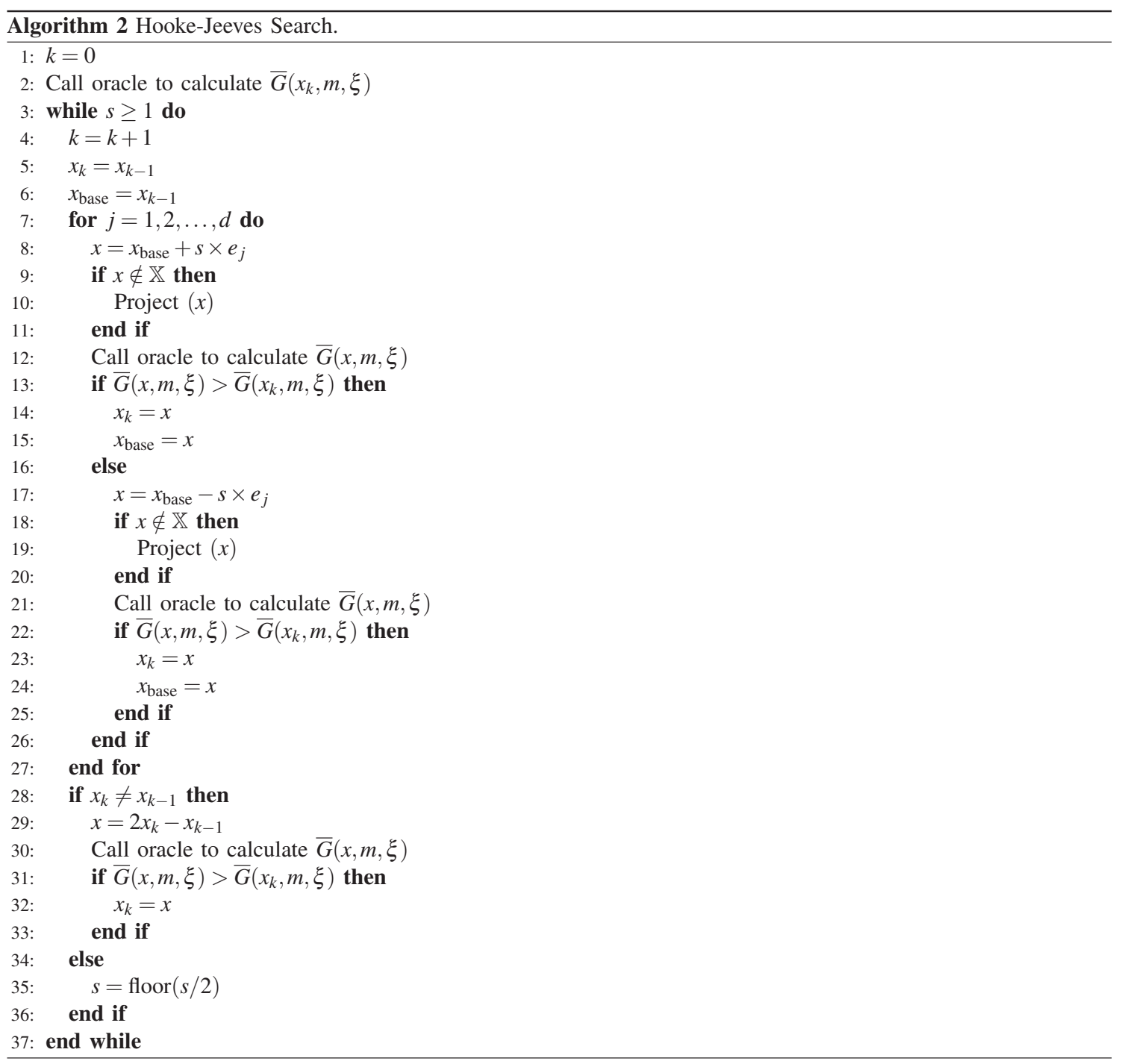

\section{NUMERICAL EXPERIMENT USING RETROSPECTIVE OPTIMIZATION}

We now seek to determine the location of five new production wells in the Brugge field model (Peters et al. 2009) under uncertainty. The three-dimensional reservoir model for all realizations consists of $139 \times 48 \times 9$ (total of 60,048 ) grid blocks, with each block of dimensions $100 \mathrm{~m} \times 100 \mathrm{~m} \times$ $6 \mathrm{~m}$. The model has five existing vertical water injection wells that fully penetrate the reservoir. Water injection is performed to maintain reservoir pressure and to sweep the oil toward production wells. The total production time is 30 years. Figure 1 shows one realization of oil saturation (saturation in this context is equivalent to volume fraction; the oil and water saturations sum to one) in the field before production.

The objective is to determine the optimal location of the five new production wells. This includes the areal $(i, j)$ locations of each well and the completion interval $\left(k_{1}, k_{2}\right)$, which defines the vertical block locations $k_{1}$ to $k_{2}$, with $k_{1} \leq k_{2}$, over which the well is open to flow. Thus the total number of optimization variables for this problem is 20 (4 variables per well).

We now discuss the stochastic nature of the problem. In the general case, many aspects of the reservoir model are uncertain. Key uncertainties include the type of geological depositional system and associated parameters, the presence and location of large-scale faults and fractures, and the spatial correlation structure of rock properties such as porosity and permeability. The parameters quantifying the efficiency of the displacement of oil by the injected water (e.g., residual oil saturation, endpoint 
Wang, Echeverría Ciaurri and Durlofsky

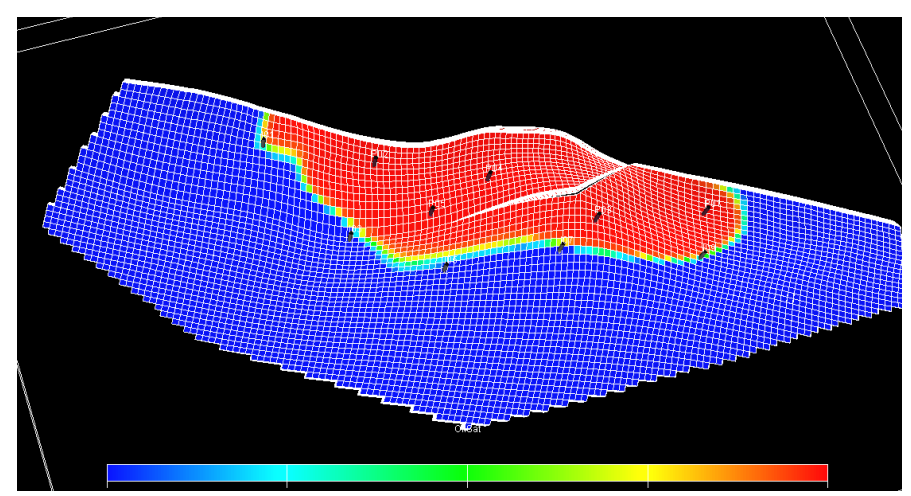

Figure 1: One realization of oil saturation in the Brugge model.

relative permeabilities), as well as the depth of the oil-water contact, can also be uncertain. In an attempt to account for these and other uncertainties, multiple realizations of the reservoir model are constructed. Depending on the number and ranges of the parameters considered, there can be tens, hundreds or thousands of model realizations. Predicted reservoir performance is then reported in terms of expected values and cumulative distribution functions for essential quantities such as oil production rate as a function of time.

In the example considered here, the uncertainty parameter includes the depth of the oil-water contact and various other geological model properties (including porosity and permeability). The 104 available realizations are assumed to be equiprobable and to adequately represent uncertainty. In a comprehensive (practical) study, it is quite possible that a larger number of uncertain parameters, and thus a larger number of realizations, would be included.

The objective function $g$ is the expected total NPV for 30 years of production over the 104 realizations of the reservoir model. The objective function is thus defined as:

$$
g=\frac{1}{104}{ }_{j=1}^{104}\left[{ }_{i=1}^{30} \frac{p_{o} q_{i, j}^{o}-p_{w_{1}} q_{i, j}^{w_{1}}-p_{w_{2}} q_{i, j}^{w_{2}}}{(1+)^{i}}\right],
$$

where $p_{o}$ is oil price $(\$ 80 / \mathrm{bbl}), p_{w_{1}}$ and $p_{w_{2}}$ are the water production and injection costs (both taken to be $\$ 5 / \mathrm{bbl}), \quad=0.0234$ is the yearly discount rate, $q_{i, j}^{o}, q_{i, j}^{w_{1}}$ and $q_{i, j}^{w_{2}}$ are the oil and water production rates and the water injection rate, respectively, for the $i^{t h}$ year and the $j^{\text {th }}$ model realization. The rates are all in units of bbl/year. Water production is a cost because equipment and energy are required to produce, separate, and dispose of water. We use the ECLIPSE ${ }^{\mathrm{TM}}$ reservoir simulator in this study. One call of $G$ gives one observation of the production quantities $q_{i, j}^{o}, q_{i, j}^{w_{1}}, q_{i, j}^{w_{2}}, i=1,2, \ldots, 30$, given an $x$ and $j$, which in turn provides one observation of $g$. It takes about 7 minutes to complete a simulation run of $G$ for a single realization of the model, running on a Windows PC with a $2.66 \mathrm{Ghz}$ $\mathrm{CPU}$ and $1.95 \mathrm{G}$ of RAM.

Our ROHJ implementation for the Brugge case generates five sample-path problems. The sequence of sample sizes $\{1,5,16,21,104\}$ is used for sample-path problems $P_{k}, k=1, \ldots, 5$, respectively. The realizations $1,2-6,7-22,23-43,1-104$ are selected for $P_{k}, k=1, \ldots, 5$, respectively. The first four sample-path problems have independent samples (no common realizations) from the sample population of 104 realizations, while the last problem $P_{5}$ includes all 104 realizations. The last sample-path problem is the true problem $P$. These problem parameters were chosen heuristically based on the available number of realizations.

Figure 2 shows five performance curves for the ROHJ algorithm for this problem. Each curve represents the search progress for one sample-path problem $P_{k}$ (with associated cost function $\bar{G}\left(x, m_{k}, k\right)$ ). Where a given curve is only partially plotted, the remainder of the curve is flat. We plot the best current solution, so the curves are monotonic in the objective function value for all $P_{k}$. The first curve involving realization 1 is steep because the given initial solution $x_{0}$ (based on heuristics) is much worse than the returned solution from $P_{1}$. The curve for $P_{3}$ is below all the others, but this does not necessarily imply that the solutions visited in $P_{3}$ are worse solutions compared to the solutions in $P_{1}$ 
and $P_{2}$ in terms of the true objective function values of $g$. It is simply the case that the realizations in $P_{3}$ yield low values of $\bar{G}\left(x, m_{3}, 3\right)$, as is evident in Figure 3, discussed next.

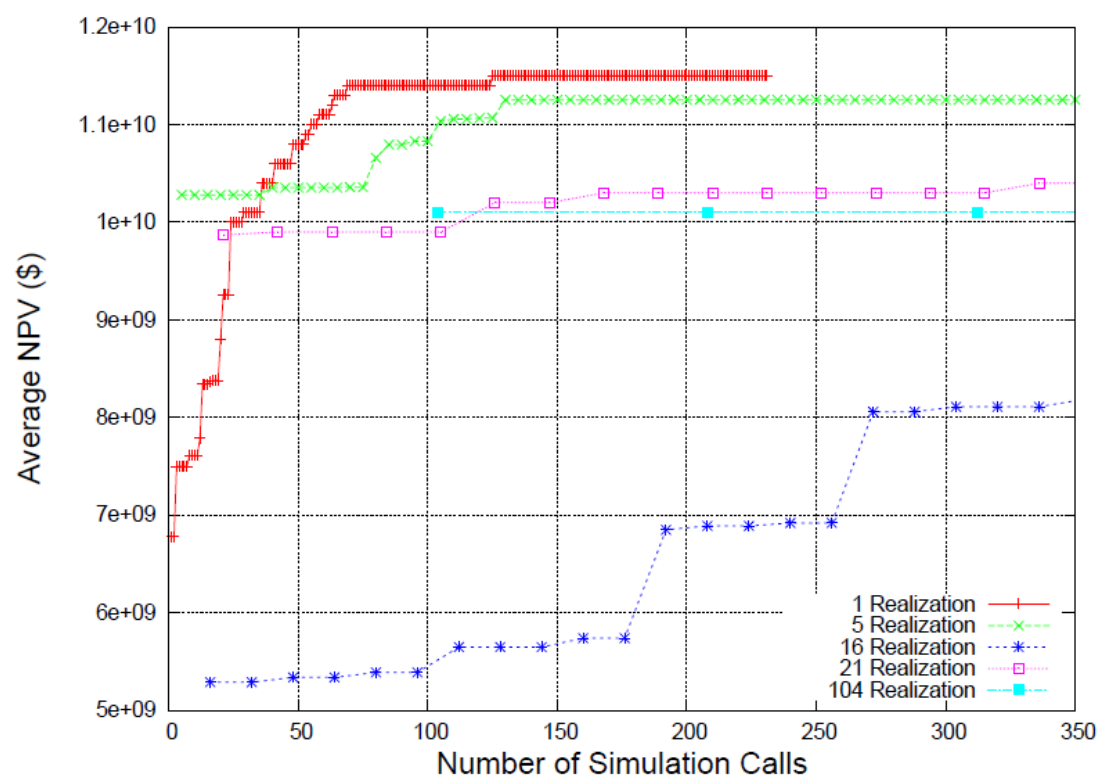

Figure 2: Performance of ROHJ for the Brugge well location problem.

We now assess the solution quality for all five sample-path problems by simulating the initial solution and the five returned solutions with all 104 realizations. Figure 3 shows that all retrospective solutions lead to improvements in $g$ except for the last one. For each solution, the box and (red) bars represent the minimum, quartiles, and the maximum NPV among the 104 observations. The (green) bar in the middle of the box is the sample mean over the 104 observations. In the last sample-path $P_{5}$, the starting solution is indeed a local optimum of $g$, thus no further improvement can be achieved. The final solution returned from $P_{5}$ yields more than a $70 \%$ increase in the average NPV over the initial solution (from $\$ 5.8$ billion to $\$ 10.1$ billion). In this particular case, RO saves about $75 \%$ of the simulations that would be required if we solved $P$ using HJ search with all 104 realizations, assuming that HJ solves $P$ by evaluating around 200 solutions (as is required for $P_{1}-$ see Figure 2), given the same initial solution $x_{0}$. Finally, we compare the oil production rates between the initial solution and final solution (returned from $P_{5}$ ) over the 30 years of production. Figure 4 shows that, for the first model realization, the optimal solution provides significant improvement in the production rates through all of the production time. Overall we can conclude that ROHJ is quite effective for this problem.

\section{CONCLUDING REMARKS}

In this work we implemented a retrospective optimization algorithm using Hooke-Jeeves direct search (ROHJ) for bounded stochastic discrete systems. The promising results for our target application optimizing the placement of oil wells in a medium-sized realistic model under uncertainty - suggest that ROHJ can be used for this class of problems to provide significant improvement in net present value. Future work will include algorithm convergence analysis and performance comparisons with other methods.

\section{ACKNOWLEDGMENTS}

The authors thank Alberto Cominelli and Andrea Luigi Lamberti of Eni S.p.A. for their valuable comments and suggestions. This research was financially supported by Eni S.p.A. 


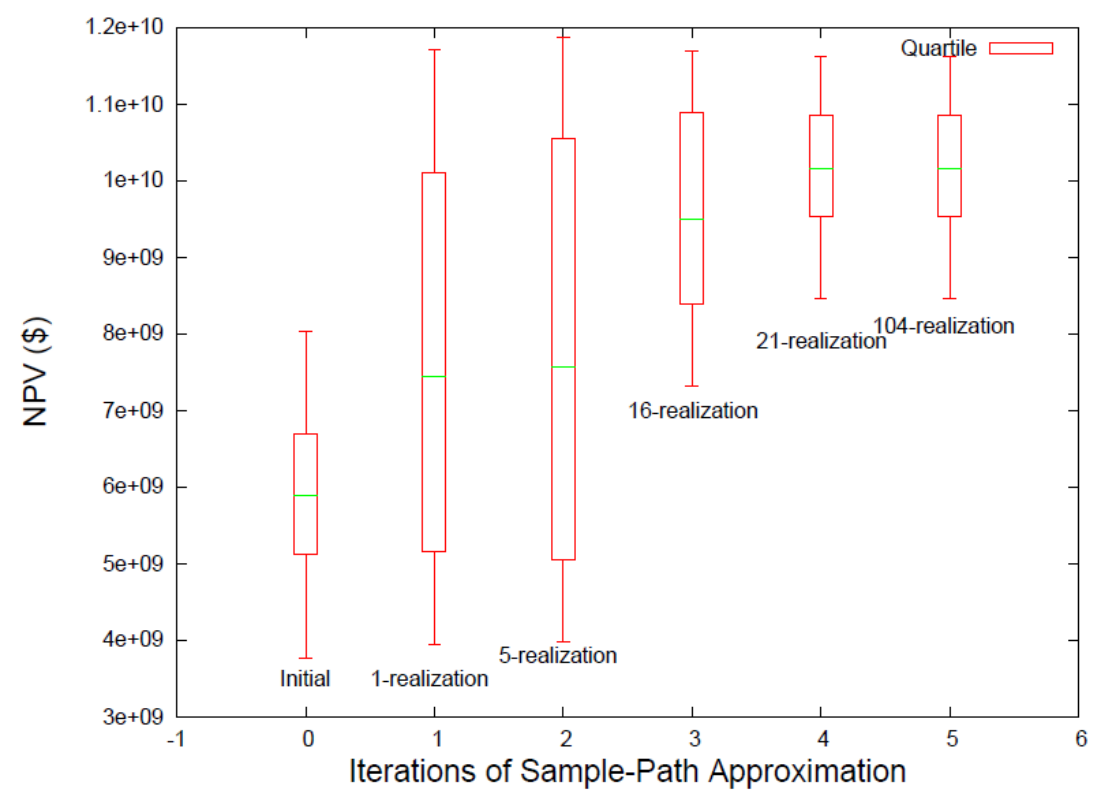

Figure 3: ROHJ solutions for the Brugge well location problem.

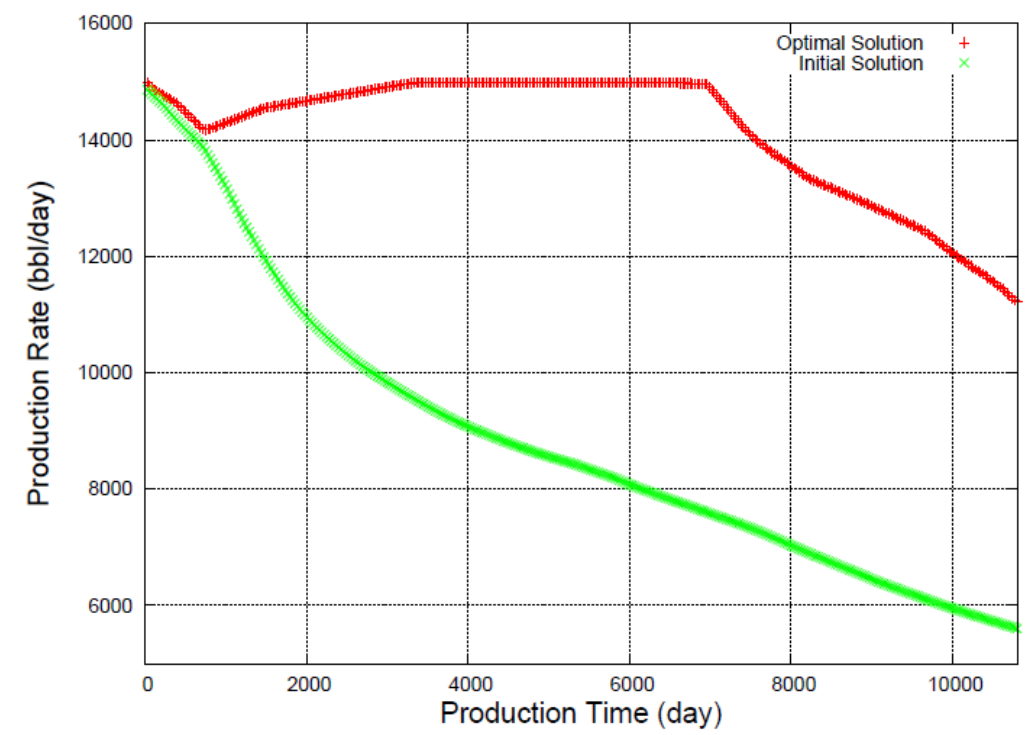

Figure 4: Comparison of oil production rate for realization 1.

\section{REFERENCES}

Bangerth, W., H. Klie, M. F. Wheeler, P. L. Stoffa, and M. K. Sen. 2006. On optimization algorithms for the reservoir oil well placement problem. Computational Geosciences 10:303-319.

Chen, H., and B. W. Schmeiser. 2001. Stochastic root finding via retrospective approximation. IIE Transactions 33:259-275. 
Hooke, R., and T. A. Jeeves. 1961. "Direct search" solution of numerical and statistical problems. Journal of the ACM 8:212-229.

Jin, J., and B. W. Schmeiser. 2003. Simulation-based retrospective optimization of stochastic systems. In Proceedings of the 2003 Winter Simulation Conference. New Orleans, Louisiana.

Kleywegt, A. J., A. Shapiro, and T. H. de Mello. 2001. The sample average approximation method for stochastic discrete optimization. SIAM Journal on Optimization 12:479-502.

Onwunalu, J. E., and L. J. Durlofsky. 2010. Application of a particle swarm optimization algorithm for determining optimum well location and type. Computational Geosciences 14:183-198.

Peters, E., R. J. Arts, G. K. Brouwer, and C. R. Geel. 2009. Results of the Brugge benchmark study for flooding optimization and history matching. In Paper SPE 119094 presented at the SPE Reservoir Simulation Symposium. The Woodlands, Texas.

Sarma, P., and W. H. Chen. 2008. Efficient well placement optimization with gradient-based algorithms and adjoint models. In Paper SPE 112257 presented at the SPE Intelligent Energy Conference and Exhibition. Amsterdam, The Netherlands.

Wang, H., and B. W. Schmeiser. 2008. Discrete stochastic optimization using linear interpolation. In Proceedings of the 2008 Winter Simulation Conference, ed. S. J. Mason, R. R. Hill, L. Mönch, O. Rose, T. Jefferson, and J. W. Fowler. Washington D.C., USA.

Yeten, B. 2003. Optimum deployment of nonconventional wells. Ph. D. thesis, Stanford University.

\section{AUTHOR BIOGRAPHIES}

Honggang Wang is a Postdoctoral Scholar in Energy Resources Engineering at Stanford University. His research interests lie in stochastic optimization, simulation modeling and analysis, and their applications in energy production. His email address is <honggang@stanford.edu>.

David Echeverría Ciaurri is a Research Associate within the Department of Energy Resources Engineering at Stanford University. His main research interests are efficient optimization and modeling. His email address is <echeverr@stanford.edu>.

Louis J. Durlofsky is Chairman and Professor in the Department of Energy Resources Engineering at Stanford University. His research interests include general reservoir simulation, production optimization, upscaling, modeling of advanced wells, and geological carbon sequestration. His email address is <lou@stanford.edu>. 